Daniele De Luca

Marco Piastra

Giovanna Chidini

Pierre Tissieres

Edoardo Calderini

Sandrine Essouri

Alberto Medina Villanueva

Ana Vivanco Allende

Marti Pons-Odena

Luis Perez-Baena

Michael Hermon

Ascanio Tridente

Giorgio Conti

Massimo Antonelli

Martin Kneyber

On behalf of Respiratory Section of the European Society for Pediatric Neonatal Intensive Care (ESPNIC)

Received: 27 May 2013

Accepted: 7 September 2013

Published online: 8 October 2013

(C) Springer-Verlag Berlin Heidelberg and ESICM 2013

Partial results were presented as a late-

breaking oral communication at the 24th

Annual Meeting of the European Society of

Pediatric and Neonatal Intensive Care,

Rotterdam (NL), June 2013.

A related editorial can be found at: doi:

10.1007/s00134-013-3094-6.

Electronic supplementary material

The online version of this article

(doi:10.1007/s00134-013-3110-x) contains

supplementary material, which is available

to authorized users.

D. De Luca (®)

Terapia Intensiva Pediatrica-DEA, Policlinico Universitario “A. Gemelli”Università Cattolica del Sacro Cuore,

L.go A. Gemelli 8, 00168 Rome, Italy

e-mail: daniele.deluca@rm.unicatt.it; dm.deluca@fastwebnet.it

Tel.: +39-6-30155203

Fax: +39-6-30155283

D. De Luca

Neonatal Intensive Care Unit, CHU Antoine Béclère, South Paris University Teaching Hospitals, Paris, France

\section{The use of the Berlin definition for acute \\ The use of the Berlin definition for acute
respiratory distress syndrome during infancy and early childhood: multicenter evaluation and expert consensus (2)}

\section{G. Chidini · E. Calderini}

Pediatric Intensive Care Unit,

Department of Anesthesia and Critical Care,

Fondazione IRCCS Ca' Granda,

Ospedale Maggiore Policlinico,

Milan, Italy

P. Tissieres - S. Essouri

Pediatric Intensive Care Unit,

University Teaching Hospitals,

Paris, France

A. Medina Villanueva .

A. Vivanco Allende

Pediatric Intensive Care Unit,

Department of Pediatrics,

Hospital Universitario de Asturias,

Oviedo, Spain

M. Pons-Odena · L. Perez-Baena Pediatric Intensive Care and Intermediate Care Unit, Department of Pediatrics, Hospital Universitari Sant Joan de Déu, Barcelona, Spain

\section{Hermon}

Division of Neonatology,

Intensive Care and Neuro-pediatrics,

Medical University of Vienna,

Vienna, Austria

\section{A. Tridente}

Intensive Care Unit, Whiston Hospital, St. Helen's and Knowsley Teaching Hospitals NHS Trust, UK and academic Unit of Medical Education, Sheffield Medical School, University of Sheffield, Sheffield, UK CHU Kremlin-Bicetre, South Paris

\section{Antonelli}

General Intensive Care Unit, Department of Anesthesia and Intensive Care, University Hospital "Agostino Gemelli", Catholic University of the Sacred Heart, Rome, Italy

M. Kneyber

Pediatric Intensive Care Unit, Department of Pediatrics, Beatrix Children's Hospital, University Medical Center Groningen, University of Groningen, Groningen, The Netherlands

D. De Luca · M. Piastra - G. Conti Pediatric Intensive Care Unit, Department of Anesthesia and Intensive Care, University Hospital "Agostino Gemelli”, Catholic University of the Sacred Heart, Rome, Italy

Respiratory Section of the European Society for Pediatric Neonatal Intensive Care (ESPNIC)

1-3 Rue de Chantepoulet, P.O. Box 1726, 1211 Geneva 1, Switzerland
Abstract Purpose: A new acute respiratory distress syndrome (ARDS) definition has been recently issued: the so-called Berlin definition (BD) has some characteristics that could make it suitable for pediatrics. The European Society for Pediatric Neonatal Intensive Care (ESPNIC) Respiratory Section started a project to evaluate $\mathrm{BD}$ validity in early childhood. A secondary aim was 
reaching a consensus on clinical tools (risk factors list and illustrative radiographs) to help the application of BD. Methods: This was an international, multicenter, retrospective study enrolling 221 children [aged greater than 30 days and less than 18 months; median age 6 (range 2-13) months], admitted to seven European pediatric intensive care units (PICU) with acute lung injury (ALI) or ARDS diagnosed with the earlier definition. Results: Patients were categorized according to the two definitions, as follows: ALI, 36;

ARDS, 185 (for the American-European Consensus Conference (AECC) definition); mild, 36; moderate, 97; severe ARDS, 88 (for BD). Mortality
(13.9\% for mild ARDS; $11.3 \%$ for moderate ARDS; $25 \%$ for severe ARDS, $p=0.04$ ) and the composite outcome extracorporeal membrane oxygenation (ECMO)/mortality (13.9\% for mild ARDS; $11.3 \%$ for moderate ARDS; $28.4 \%$ for severe ARDS, $p<0.01$ ) were different across the BD classes, whereas they were similar using the previous definition. Mortality [HR 2.7 (95\% CI 1.1-7.1)] and ECMO/mortality [HR 3 (95\% CI 1.1-7.9)] were increased only for the severe ARDS class and remained significant after adjustment for confounding factors. PICU stay was not different across severity classes, irrespective of the definition used. There was significant concordance between raters evaluating radiographs [ICC $0.6(95 \% \mathrm{CI}$ $0.2-0.8)$ ] and risk factors [ICC 0.92 (95\% CI 0.8-0.97)]. Conclusions: BD validity for children is similar to that already reported in adults and mainly due to the introduction of a "severe ARDS" category. We provided clinical tools to use BD for clinical practice, research, and health services planning in pediatric critical care.

\section{Keywords ARDS · Children ·}

Diagnostic criteria

\section{Introduction}

The American-European Consensus Conference (AECC) defined acute respiratory distress syndrome (ARDS) 19 years ago [1]: since then, this definition has not been modified and has been used worldwide for clinical and research purposes. In the meantime, accumulating data led to criticisms of the AECC definition, and thus ARDS has been recently redefined according to an international consensus endorsed by various scientific societies [2]. The new Berlin definition (BD) addresses the criticisms about feasibility, reliability, and validity of the AECC definition [3]. For instance, invasive measurement of pulmonary pressure has been removed from the diagnostic criteria and the use of echocardiography to exclude increased left atrial pressures has been advocated; clinical severity has been divided into three categories defined by the oxygenation deficit; a minimum level of positive end-expiratory pressure (PEEP) has been included in the definition; clinical and radiological examples have been created in order to increase interobserver agreement and help in identifying the syndrome $[2,3]$.

The AECC definition had been applied to pediatric ARDS without specific evaluations or changes [4-6]. However, ARDS is a complex syndrome: any definition is an attempt to categorize the clinical reality and this complexity is partially due to the different underlying conditions and triggering diseases across the ages [7]. Moreover, pediatric ARDS shows differences in epidemiology, prognosis, and management. These differences especially occur during early pediatric age [7]. Given these dissimilarities, a new ARDS definition should be specifically evaluated for pediatric patients.
BD has been issued through a formal consensus process, followed by an empirical evaluation over a large cohort of patients coming from different databases, demonstrating that the BD predicts mortality and ventilatory requirements better than the AECC definition [2]. Finally, illustrative clinical pictures for ARDS identification have also been produced.

The Respiratory Section of the European Society for Pediatric and Neonatal Intensive Care (ESPNIC) considered mandatory a formal evaluation of a clinical definition before applying it to pediatric patients. Thus, we followed the same process already used for adults [2] and our main goal was to investigate the validity of BD in infancy and early childhood. Our secondary aim was to develop a consensus on some clinical tools, namely a risk factors list and illustrative radiographs, to help the application of BD in infancy and early childhood [3]. Results were partially presented as a late-breaking communication at the 24th ESPNIC Meeting [8].
Methods
Working group and study protocol
Members of the ESPNIC Respiratory Section were asked to participate in a validation of BD during infancy and early childhood. Inclusion criteria were the availability of institutional electronic databases of patients admitted to pediatric intensive care units (PICU), where ARDS was diagnosed according to the AECC definition [2]. Data were recorded in real time and searched retrospectively. Seven academic PICUs declared their interest and 
fulfilled the inclusion criterion. A member (M.A.) of the ARDS Definition Task Force that originally issued the $\mathrm{BD}$ was also asked to join the project and thus a specific working group was created. A group coordinator (D.L.D.) was in charge for the organization of meetings, communication, and the further steps of the project.

The working group and the ESPNIC Respiratory Section members had serial communications by mailshot or teleconference that were supported by the ESPNIC administrative secretariat. Within the working group, an informal Quaker-based consensus technique was used [9].
Consensus was demonstrated by unanimity; in case of disagreement, all investigators had the opportunity to discuss and reach an agreement; otherwise individual votes were counted.

The study outline is reported in Fig. 1. The working group had preliminary discussions about the project outline: it was decided to test BD in early childhood with no changes, because some features of the definition have been unanimously recognized as suitable for pediatric critical care (Table 1). The institutional review board at the Catholic University of the Sacred Heart, Department
Fig. 1 Outline of the project. Both the BD evaluation and the production of clinical tools are described. Phases performed at the coordinating center and those in each participating center (or by ESPNIC Respiratory Section members) are in black and gray, respectively

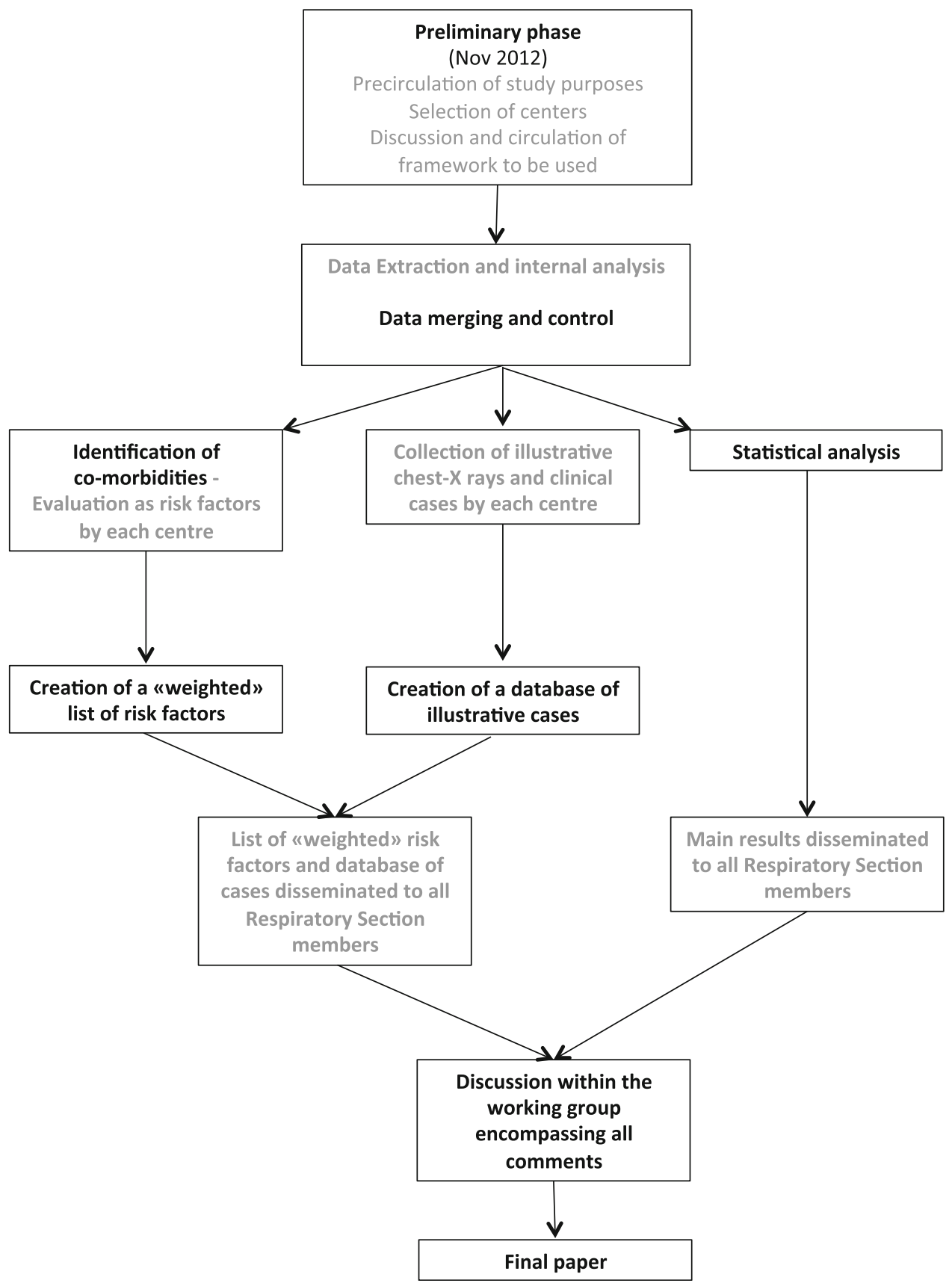


Table 1 New Berlin definition (modified from [2,3]) and reasons for its theoretical suitability in infancy and early childhood

\begin{tabular}{|c|c|c|}
\hline \multicolumn{2}{|c|}{ Berlin definition criteria } & \multirow{2}{*}{$\begin{array}{l}\text { Suitability in infants } \\
\text { Acute time frame is specified }\end{array}$} \\
\hline Timing & $\begin{array}{l}\text { Within } 1 \text { week of a known clinical insult or } \\
\text { new or worsening respiratory symptoms }\end{array}$ & \\
\hline $\begin{array}{l}\text { Chest X-rays or } \\
\text { tomography scan }\end{array}$ & $\begin{array}{l}\text { Bilateral opacities not fully explained by effusions, } \\
\text { lobar/lung collapse, or nodules. (Illustrative } \\
\text { clinical cases and chest X-rays have been provided) }\end{array}$ & $\begin{array}{l}\text { Including illustrative radiographs is important, } \\
\text { because ARDS appearance may be different } \\
\text { in children and in adults }\end{array}$ \\
\hline Origin of edema & $\begin{array}{l}\text { Respiratory failure not fully explained by } \\
\text { cardiac failure or fluid overload. Need } \\
\text { objective assessment (e.g., echocardiography) } \\
\text { to exclude hydrostatic edema, if no ARDS risk } \\
\text { factors are present }\end{array}$ & $\begin{array}{l}\text { Echocardiography widely used, whereas } \\
\text { Swan-Ganz catheters are rarely used in } \\
\text { early childhood. Including risk factors in } \\
\text { the ARDS definition is important, because } \\
\text { they may be different in children and } \\
\text { in adults }\end{array}$ \\
\hline \multicolumn{3}{|l|}{ Oxygenation $^{\mathrm{a}}$} \\
\hline Mild & $\begin{array}{l}200 \mathrm{mmHg}<\mathrm{PaO}_{2} / \mathrm{FIO}_{2} \leq 300 \mathrm{mmHg}^{\mathrm{mHg}} \\
\text { with PEEP or CPAP }>5 \mathrm{cmH}_{2} \mathrm{O}^{\mathrm{b}}\end{array}$ & \multirow{3}{*}{$\begin{array}{l}\text { Noninvasive CPAP is widely used in early } \\
\text { childhood. PEEP threshold }\left(5 \mathrm{cmH}_{2} \mathrm{O}\right) \text { is a } \\
\text { value commonly used during early } \\
\text { childhood }\end{array}$} \\
\hline Moderate & $\begin{array}{l}100 \mathrm{mmHg}<\mathrm{PaO}_{2} / \mathrm{FIO}_{2} \leq 200 \mathrm{mmHg} \\
\text { with } \mathrm{PEEP}>5 \mathrm{cmH}_{2} \mathrm{O}\end{array}$ & \\
\hline Severe & 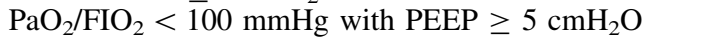 & \\
\hline
\end{tabular}

CPAP continuous positive airway pressure, $\mathrm{FIO}_{2}$ fraction of inspired oxygen, $\mathrm{PaO}_{2}$ partial pressure of arterial oxygen, PEEP positive end-expiratory pressure

${ }^{\text {a }}$ If altitude is higher than $1,000 \mathrm{~m}$, the correction factor should be calculated as follows: $\left[\mathrm{PaO}_{2} / \mathrm{FIO}_{2} \times(\right.$ barometric pressure/760)]

of Anesthesia and Intensive Care, approved the study and waived the need for informed consent.

\section{Variables}

Patients diagnosed with acute lung injury (ALI) or ARDS according the AECC definition [1] and without congenital heart diseases or major congenital malformations were identified. We focused on the early pediatric age because it is considered to carry underlying clinical conditions different from the adult population and because of the particular mechanical and developmental situation of the chest and lung [10]. An age cutoff has never been used in this regard; thus the working group discussed and unanimously agreed to consider only patients aged greater than 30 days and less than 18 months.

The following data were anonymously extracted from the databases of participating PICUs for the years 2009-2011: age and weight on admission, sex, PRISM$\mathrm{III}_{24}$ [11], $\mathrm{PaO}_{2} / \mathrm{FiO}_{2}$ at the ARDS diagnosis [12], and type of ARDS (primary or direct ARDS, due to a primarily pulmonary condition; secondary or indirect ARDS, due to a systemic or primarily non-pulmonary condition). All data were kept secure following local regulations.

Our hypothesis was that BD describes the clinical reality of ARDS in infants and small children better, because of its intrinsic features. Thus, PICU mortality and the composite variable PICU need for extracorporeal membrane oxygenation (ECMO)/mortality were chosen as main outcomes. Survival time and survival time without ECMO (ECMO-free survival time) have b This may be delivered noninvasively in the mild acute respiratory distress syndrome group

consequently been considered. PICU length of stay for survivors was a secondary outcome.

There was no common protocol between the centers, but ECMO was generally started when oxygenation index $[\mathrm{OI}=($ mean airway pressure $\times$ inspired oxygen fraction $\left.\times 100) / \mathrm{PaO}_{2}\right)$ ] was above 40 or if central venous saturation was below $65 \%$ and blood lactate above $2 \mathrm{mmol} / \mathrm{L}$. These parameters had to persist for $6-8 \mathrm{~h}$ despite maximal respiratory support and optimal general care.

Minute ventilation at the worst blood gas analysis standardized for $\mathrm{PaCO}_{2}=40 \mathrm{mmHg}\left[\left(\mathrm{VE}_{\text {corr }}\right)=\right.$ minute ventilation $\times$ worst $\left.\mathrm{PaCO}_{2} / 40\right)$ ] was also available for a single PICU. $\mathrm{VE}_{\text {corr }}$ was added to the $\mathrm{BD}$ to define severe ARDS and tested, as previously published [2]. Bronchopulmonary dysplasia (BPD) was diagnosed according to the National Institute for Child Health and Human Development definition [13].

A unique data sheet was used to extract data and each center performed an internal validity analysis, reviewing all patients' files and chest X-rays, as well. Two investigators for each center independently reviewed data: all patients diagnosed with the original AECC definition met the BD criteria, as well.

Data were sent to the coordinating center, where they have been merged and checked. Cases were stratified according to the clinical severity. To do this, we used the three BD categories and considered the AECC definition dividing cases into two categories [2]: ARDS $\left(\mathrm{PaO}_{2} /\right.$ $\left.\mathrm{FiO}_{2} \leq 200\right)$ and ALI non-ARDS $\left(200 \mathrm{mmHg}<\mathrm{PaO}_{2} /\right.$ $\mathrm{FiO}_{2} \leq 300$ ). Results were sent to ESPNIC Respiratory Section members to receive their comments that were 
considered by the working group for the final manuscript (see Fig. 1).

\section{Clinical tools (risk factors and illustrative radiographs)}

All comorbidities for each patient were identified and a complete list was circulated within the working group. Investigators were asked to estimate the relevance of each risk factor for ARDS development, using a linear scoring system ( $0=$ minimum; $5=$ maximum $)$. Relevance of a variable was considered both as its importance for the pathogenesis of pediatric ARDS and as its frequency amongst pediatric ARDS patients. Evaluations of each investigator were averaged and a "weighted" list of risk factors was created. Variables with a mean score of less than 1 were considered unimportant and excluded. Comorbidities were originally reported as free text and then transformed for the analysis following a unique standardized code. Each investigator was also asked to provide in detail illustrative chest X-rays and clinical data for some cases. These were discussed within the working group in order to create a database of illustrative clinical cases. The working group agreed on a data set of 12 cases describing typical pediatric situations.

Risk factors and radiographs were then sent to the members of ESPNIC Respiratory Section for a blind evaluation. The members were asked to evaluate each risk factor using the same score and to evaluate chest X-rays as "consistent"/"equivocal"/"non-consistent" for ARDS [2]. This ensured that the evaluation done by the working group was not subjective but concordant with the judgment given by a large number of colleagues with expertise in the respiratory field (see Fig. 1).

\section{Statistics}

Continuous variables (such as $\mathrm{PaO}_{2} / \mathrm{FiO}_{2}$ or weight) were tested for normality using the Kolmogorov-Smirnov test and described accordingly as mean [95\% confidence interval (CI)] or median (interquartile range). PICU stay in survivors was analyzed across the different ARDS severity classes using the Kruskal-Wallis $H$ test, because it was not normally distributed.

Univariate Cox's regression was used as the univariate analysis for the main outcomes (mortality and ECMO/ mortality) and gave the hazard ratios [HR (95\% CI)]. Then, multivariate Cox's regressions were performed for the same outcomes adjusting for age, sex, PRISM-III ${ }_{24}$, ARDS type (primary/secondary), and study center. These covariates were chosen because of their epidemiological role $[4,14]$ and their association with the outcomes [11, $15,16]$ by unanimous agreement within the working group. BD definition was inserted in the model and considered to have three categories. Cox's multivariate regressions were performed with a backward-stepwise method: a covariate was removed from the model if its significance in the Cox's model was $p>0.10[16,17]$. The likelihood ratio test was used to estimate the model goodness-of-fit [16, 17].

Kaplan-Meier analysis was also performed and the Breslow test was used to compare curves, according to the hypothesis that all patients discharged alive from the PICU were alive at day 40. Receiver operator characteristic curve (ROC) analysis was applied, as well. Areas under the ROC curves (AUC) were compared with De Long's technique [18]. Consistency of risk factors and radiograph evaluation amongst raters were analyzed by intraclass correlation coefficients (ICC) for averaged measures under a mixed effect model [19]. Analyses were conducted with SPSS for Windows release 15 (SPSS inc, Chicago, IL, USA), MedCalc for Windows release12.1 demo (MedCalc Software, Ostend, Belgium).

\section{Results}

Evaluation of Berlin definition in pediatric ARDS patients

A total of 221 patients were identified as eligible and were all enrolled in the study after review of their files. Demographics and basic data of the studied population are shown in Table 2. Four $(1.8 \%)$ patients needed ECMO. Infants were cared for in seven PICUs from Italy, Spain, France, Austria, and the Netherlands.

Table 3 and Fig. 2 show the univariate analyses of outcomes stratified according to ARDS severity using AECC and BD criteria. The risk for mortality [HR for moderate ARDS, 0.8 (95\% CI 0.3-2.5), $p=0.77$; HR for severe ARDS, 2.7 (95\% CI 1.1-7.1), $p=0.04$ ] and

Table 2 Characteristics of the studied population

\begin{tabular}{ll}
\hline Patients $(n)$ & 221 \\
Basic data & $6(2-13)$ \\
Age (months) & $159(71.9)$ \\
$<6$ & $49(22.1)$ \\
$\geq 6$ and $<12$ & $13(5.9)$ \\
$\geq 12$ & $7.5(4.5-15)$ \\
Weight $(\mathrm{kg})$ & $111(51.1)$ \\
Male sex & $14.6(95 \%$ CI 1-28.2) \\
${\mathrm{PRISM}-\mathrm{III}_{24}}_{\mathrm{PaO}_{2} / \mathrm{FiO}_{2} \text { at the diagnosis }}$ & $133(95 \%$ CI 3-262) \\
$\mathrm{Primary} \mathrm{ARDS}_{\text {Outcomes }}$ & $89(40.3)$ \\
Mortality & $38(17.2)$ \\
Mortality/ECMO & $41(18.6)$ \\
PICU stay (days) & $10(6-16)$ \\
\hline
\end{tabular}

Data are given as number $(\%)$, mean $(95 \% \mathrm{CI})$, or median (interquartile range), as appropriate

$E C M O$ extracorporeal membrane oxygenation, $P I C U$ pediatric intensive care unit, $P R I S M-I I I_{24}$ pediatric risk index for mortality 
Table 3 Distribution of the main outcomes across categories of the AECC and Berlin definitions

\begin{tabular}{|c|c|c|c|c|c|c|}
\hline \multicolumn{3}{|l|}{ AECC definition } & \multicolumn{4}{|l|}{ Berlin definition } \\
\hline Category $(n)$ & ALI non-ARDS (36) & ARDS (185) & Category $(n)$ & Mild (36) & Moderate (97) & Severe $(88)$ \\
\hline Mortality & $5(13.9)$ & $33(17.8)$ & Mortality & $5(13.9)$ & $11(11.3)$ & $22(25)$ \\
\hline ECMO/mortality & $5(13.9)$ & $36(19.5)$ & ECMO/mortality & $5(13.9)$ & $11(11.3)$ & $25(28.4)$ \\
\hline
\end{tabular}

Data are given as raw number (\%). Risk for mortality and ECMO/ mortality was significantly increased only for the severe ARDS category of the Berlin definition (see text for details)
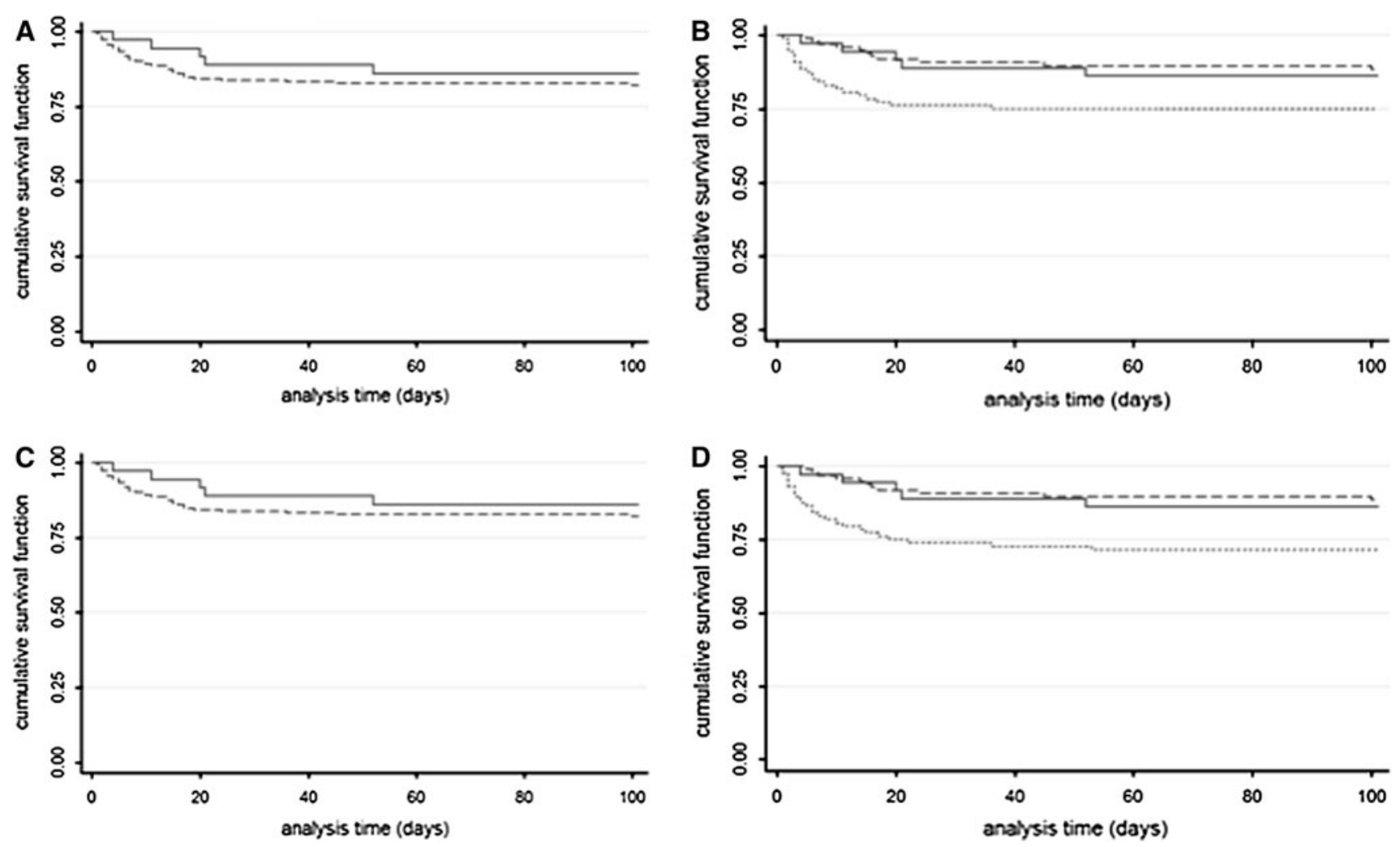

Fig. 2 Kaplan-Meyer curves for main outcomes according AECC and BD classes of severity. Survival according to the AECC (a) and Berlin (b) definitions. Survival without the need for ECMO according to the AECC (c) and Berlin (d) definitions. In a and c, solid and dashed lines represent ALI non-ARDS and ARDS categories, respectively. In $\mathbf{b}$ and $\mathbf{d}$, solid, dashed, and dotted lines

for the composite outcome $\mathrm{ECMO} /$ mortality $[\mathrm{HR}$ for moderate ARDS, 0.8 (95\% CI 0.3-2.4), $p=0.71$; HR for severe ARDS, 3 (95\% CI 1.1-7.9), $p=0.02$ ] were significantly increased only for BD "severe ARDS" class. Risks were not significantly increased across AECC classes (data not shown). Kaplan-Meier analysis showed the following median survival times: 52 (21-52) days (mild ARDS), 100 (24-100) days (moderate ARDS), and 36 (11-36) days (severe ARDS) across BD severity classes; 52 (21-52) days (ALI non-ARDS) and 52 (20-100) days (ARDS) across AECC categories. Mean ECMO-free survival time was $52(21-52)$ days (mild

$A L I$ acute lung injury, $A R D S$ acute respiratory distress syndrome, $E C M O$ extracorporeal membrane oxygenation

represent mild, moderate, and severe ARDS, respectively. Significant differences are shown: $* p=0.04 ; \# p=0.025$. Curves were not significantly different across AECC categories (survival, $p=0.32$; survival without ECMO, $p=0.26$ ); $p$ values are given by the Breslow's test

ARDS), 100 (24-100) days (moderate ARDS), and 22 (11-36) days (severe ARDS) across BD severity classes, whereas it was 52 (21-52) days (ALI non-ARDS) and 53 (17-100) days (ARDS) across AECC categories.

Multivariate analyses confirmed the results for the severe ARDS class [HR for mortality, 3.8 (95\% CI 1.5-9.5), $p<0.01$; HR for ECMO/mortality, 3.4 (95\% CI 1.4-8.4), $p<0.01]$; model goodness-of-fit was satisfactory $(p=0.338$ and $p=0.256$, for the two models, respectively).

PICU stay for survivors was not significantly different across the different categories of both definitions (ALI 
Table 4 Risk factors for ARDS in infancy and early childhood

\begin{tabular}{ll}
\hline Risk factors & Score \\
\hline Sepsis & 4.4 \\
Near-drowning & 3.9 \\
Congenital immunodeficiencies & 3.8 \\
Thoracic trauma & 3.7 \\
Flu & 3.4 \\
Pneumonia-lower respiratory tract infection & 3.3 \\
Bronchiolitis & 3.3 \\
Pertussis & 2.9 \\
BPD & 2.6 \\
Pediatric cancer & 2.6 \\
Milk aspiration & 2.4 \\
Major surgery & 1.7 \\
Gastroesophageal reflux & 1.7
\end{tabular}

Figures are the average of a semiquantitative score given by working group members to each comorbidity present in the whole data set. The score indicates the relevance attributed to each factor by the raters. Relevance was considered both as its importance for the pathogenesis of pediatric ARDS and as its frequency amongst pediatric ARDS patients. Investigators were asked to evaluate the relevance of each comorbidity, according to a linear scoring system $(0=$ minimum; $5=$ maximum $)$. These risk factors are substantially different from those published along with the original Berlin definition for adult patients [2]

$B P D$ bronchopulmonary dysplasia

non-ARDS, 12 (6-21); ARDS, 10 (6-15) days; $p=0.108$ for AECC. Mild, 12 (6-21); moderate, 11 (7.8-15); severe, 8 (4-17) days; $p=0.09$ for $\mathrm{BD}$ ).

ROC analyses testing the predictive validity of ARDS definitions for the main outcomes are shown in the Electronic Supplementary Material 1.

\section{Clinical tools (risk factors and illustrative radiographs)}

Table 4 presents the ARDS risk factors having a mean relevance score greater than 1 , as estimated by the working group. This list was blindly evaluated by 20 members of the ESPNIC Respiratory Section with similar results and high concordance between raters [ICC 0.92 (95\% CI 0.8-0.97)].

A data set of clinical cases is also provided in the Electronic Supplementary Material 2 with advice for chest X-ray interpretation. Briefly, patients with a clinical syndrome compatible with ARDS are likely to show radiologic features from clear cardiogenic edema to clear ARDS, with many gray cases in the middle. However, pure cardiogenic edema is uncommon in infancy and early childhood in comparison to adults, except for patients affected by congenital heart diseases. Moreover, chest X-ray interpretation may be complicated by some pediatric peculiarities (presence of thymus or particular conditions like BPD), whereas others are much rarer in infancy and early childhood than in adulthood (e.g., obesity). Radiographs were blindly evaluated by 20 members of the ESPNIC Respiratory Section: there was a fair concordance in chest $\mathrm{X}$-ray evaluations between raters [ICC $0.6(95 \%$ CI 0.2-0.8)].

\section{Discussion}

If a new ARDS definition is thought to be suitable for pediatric critical care and would really prove to be reliable in this particular setting, this definition would be a useful tool to better describe the clinical reality and improve clinical assistance and research. This is the case with BD, because it improves the classification of ARDS in early childhood, describing its clinical picture [20]. This project was needed because, despite the original ARDS description included children [21], ARDS definitions have never been evaluated in multicenter pediatric populations. The early pediatric age is a relatively homogeneous population where ARDS may be more different from the syndrome observed in adults. The first difficulty was the definition of the time frame, as this was the first study in this regard. Age thresholds may be obviously criticized and the 18-months cutoff was chosen to include infants and young toddlers. This is clearly a compromise, but it was agreed because these patients are thought to carry underlying conditions and triggering diseases particularly different from adults. Furthermore, the respiratory tree is still under maturational development and this might also influence the clinical expression [7]. Practically, we wanted to test the predictive validity of the BD under extreme conditions. This meant testing $\mathrm{BD}$ against the most stringent conditions (i.e., the most relevant age range with distinct characteristics). This adds significantly to the relevance of the data, as these peculiarities lead to differences in terms of epidemiology, prognosis, ventilatory modalities [7], efficacy of surfactant [15, 22], and ancillary therapies [23], as well.

Our project allowed the collection of data from seven major academic European PICUs that ensure comparable patient care. Data accuracy was also high, being ensured by the existence of local electronic databases and the review of all clinical files. As shown in Fig. 1, an iterative and definite algorithm was used; a small working group was in charge of data analysis or interpretation and the identification of risk factors and illustrative clinical vignettes.

The used data set was fairly representative of the clinical reality: general data and outcomes are consistent with those previously published $[7,24,25]$. BD seems to describe the clinical situation better than the AECC definition achieving results similar to those reported in adults [2]. Our population is quite fairly distributed across BD categories and the predictive validity is mainly due to the introduction of a new "severe ARDS" category that helps in describing the clinical spectrum of ARDS. In fact, the main outcomes were significantly different only for severe ARDS, which carries an adjusted risk for PICU 
mortality or ECMO/mortality of 3.8 and 3.4 times higher than the mild ARDS, respectively. Clearly, ECMO cases occurred only in the severe ARDS class. PICU stay was not different across severity classes, irrespective of the definition used: this may reflect the various underlying diseases and comorbidities that may impact on PICU stay.

Looking at the ROC analysis (Electronic Supplementary Material 1), results are very similar to the original evaluation in adults [2] and, likewise, the addition of $\mathrm{VE}_{\text {corr }}$ did not improve the identification of high-risk patients [2]. AECC criteria could not accurately reflect either raw mortality or ECMO/mortality.

The mild ARDS class in the BD corresponds to the earlier ALI non-ARDS category. There are no differences between mild and moderate classes, but the presence of a severe ARDS category increases the BD validity. Thus, the new ARDS definition seems tailored to define the syndrome in infancy and early childhood, subdividing it into mild/moderate and severe ARDS. Moreover, some BD criteria (Table 1) may also account for this suitability. For instance, the 5- $\mathrm{cmH}_{2} \mathrm{O}$ PEEP threshold may be relevant for pediatric patients known to have smaller lungs and greater tendency to collapse, as compared to adults. Moreover, owing to the uncommon use of pediatric Swan-Ganz catheters, our patients were all subjected to echocardiography and this excluded any cardiogenic lung edema.

We applied a scoring system to classify ARDS risk factors and we tried to be as comprehensive as possible, starting from all comorbidities reported in our population. Then, the main risk factors were evaluated by a larger number of clinicians to estimate their relevance. Consistent with a previous report [7], several risk factors (Table 4) were infections (sepsis, flu, lower respiratory tract infections, bronchiolitis, pertussis) or conditions favoring infections (congenital immune deficiencies). The risk factors list will be useful to reasonably exclude cardiogenic lung edema and fulfill the diagnosis of pediatric ARDS.

A data set of clinical vignettes and radiographs (Electronic Supplementary Material 2) was also important because the chest $\mathrm{X}$-ray criterion is known to have moderate interobserver reliability, as confirmed by our ICC coefficient [26]. However, this may be improved through the routine use of a training set of radiographs [26-28] and thus this part of our project will help the bedside application of BD.

We acknowledge some study limitations. Our study followed the outline of the original Berlin project and thus shows some degree of subjectivity for the issues subjected only to experts' opinion. Our population is relatively small when compared to that used for the original $\mathrm{BD}$ evaluation [2]. Because ARDS is much less frequent in children than in adults [7], it is not possible to include huge populations and our study is underpowered for mortality (although power for ECMO/mortality has been estimated around $60 \%$ ). Furthermore, we based the project only on the multicenter evaluation of existing PICU databases, because very few trials have been conducted for pediatric
ARDS, as mortality is not a feasible endpoint [7]. Thus, it was not possible to create a large cohort of data from trialed children. Moreover, further specific investigations may be warranted for other pediatric ages.

Moreover, our study was not specifically targeted to identify risk factors and the strength of their association to ARDS, but rather to suggest, through an expert consensus, which variables might be ARDS risk factors. This was needed, as the underlying conditions are basically different between adulthood and early childhood. Clearly, a different study design, including control group of nonARDS infants, should be followed to verify the strength of association for each ARDS risk factor. Risk factors were evaluated with semiquantitative scoring and our study added the subjectivity of one expert group, but this does not lead to complete objectivity. Nonetheless, because all comorbidities were evaluated, an idea of the relative importance of these clinical conditions is given.

Also, our data could not be evaluated for the presence of "histological" ARDS, as lung biopsy is uncommon in children. Nor was lung histology included in the original $\mathrm{BD}$ evaluation because it is not the only pathological correlate of ARDS [3, 29]; however, in a recent study using BD, diffuse alveolar damage was more frequently observed with increasing ARDS severity [30].

We focused on PICU mortality rather than hospital mortality because of the diffuse regionalization of pediatric critical care. This was decided to avoid introduction of biases and data loss that are less frequent in adult critical care. However, ARDS definitions are generally not useful for prognostication of clinical outcomes, whereas many predictive scores [11] are available and show higher predictive validity.

Finally, the difference in AUCs between the two definitions is not huge and is comparable to what has been reported in adults [2]. However, ARDS definition is not a predictive tool, whereas other variables, which are not included in the definition, might be used more efficaciously to predict mortality in PICU [7, 11, 24, 25]. In fact, we have to keep in mind that AUC does not consider death or ECMO/death as time-dependent variables. The ROC curve is an ancillary analysis and considering a twoor three-class variable cannot be useful from a prognostic point of view for a single patient.

\section{Conclusions}

We demonstrated the validity of BD in infancy and early childhood. The good performance of BD seems to be mainly related to the introduction of a "severe ARDS" category. Following the approach already used for adults, we also developed a consensus about clinical tools to help the application of $\mathrm{BD}$ at the bedside. BD must not to be thought of as a prognostic tool, but it may be useful for 
optimizing clinical assistance, research, and health services planning in pediatric critical care.

Acknowledgments This work was supported by the administrative secretariat of the European Society for Pediatric and Neonatal Intensive Care (ESPNIC): in detail, we thank Mrs. Anastasja Mlotek-Soulé for her technical assistance. We are also grateful to the ESPNIC President, Peter C. Rimensberger (MD), for critical review of the manuscript. We also thank all members of the ESPNIC Respiratory Section that participate at various levels in this project.

Conflicts of interest The authors declare that they have no conflict of interest. The study has no sponsor or funding.

\section{References}

1. Bernard GR, Artigas A, Brigham KL, the Consensus Committee AmericanEuropean Consensus Conference on ARDS et al (1994) Definition, mechanisms, relevant outcomes and clinical trial coordination. Am J Respir Crit Care Med 149:818-824

2. ARDS definition task force (2012) Acute respiratory distress syndrome: the Berlin definition. JAMA 307:2526-2533

3. Ferguson ND, Fan E, Camporota L et al (2012) The Berlin definition of ARDS: an expanded rationale, justification, and supplementary material. Intensive Care Med 38:1573-1582

4. Yu WL, Lu ZJ, Wang Y, Collaborative Study Group of Pediatric Respiratory Failure et al (2009) The epidemiology of acute respiratory distress syndrome in pediatric intensive care units in China. Intensive Care Med 35:136-143

5. Bindl L, Dresbach K, Lentze MJ (2005) Incidence of acute respiratory distress syndrome in German children and adolescents: a population-based study. Crit Care Med 33:209-312

6. Randolph AG, Vaughn F, Sullivan R, Pediatric Acute Lung Injury and Sepsis Investigator's Network and the National Heart, Lung, and Blood Institute ARDS Clinical Trials Network et al (2011) Critically ill children during the 2009-2010 influenza pandemic in the United States. Pediatrics 128(6):e1450 e1458

7. Randolph AG (2009) Management of acute lung injury and acute respiratory distress syndrome in children. Crit Care Med 37:2448-2454

8. De Luca D, Piastra M, Chidini G et al (2013) The new "Berlin Definition" of acute respiratory distress syndrome: clinical evaluation in infants and expert consensus. Intensive Care Med 39(suppl 1):S197-S198

9. Quaker Foundations of Leadership (1999) A comparison of Quaker-based consensus and Robert's rules of order. Earlham College, Richmond

10. Nicolai T (2006) The physiological basis of respiratory support. Paediatr Resp Rev 7:97-102
11. Pollack MM, Patel KM, Ruttimann UE (1996) PRISM-III: an updated pediatric risk of mortality score. Crit Care Med 24:743-752

12. Flori HR, Glidden DV, Rutherford GW, Matthay MA (2005) Pediatric acute lung injury: prospective evaluation of risk factors associated with mortality. Am J Respir Crit Care Med 171:995-1001

13. Jobe AH, Bancalari E, NICHD/NHLBI/ ORD Workshop Summary (2001) Bronchopulmonary dysplasia. Am J Respir Crit Care Med 163:1723-1729

14. Hu X, Qian S, Xu F, Chinese Collaborative Study Group for Pediatric Respiratory Failure et al (2010) Incidence, management and mortality of acute hypoxemic respiratory failure and acute respiratory distress syndrome from a prospective study of Chinese paediatric intensive care network. Acta Paediatr 99:715-721

15. Willson DF, Chess PR, Notter RH (2008) Surfactant for pediatric acute lung injury. Pediatr Clin North Am 55:545-575

16. Draper N, Smith H (1981) Applied regression analysis, 2nd edn. Wiley, New York

17. Norusis M (2004) SPSS 13.0 advanced statistical procedures companion. Prentice Hall, Upper Saddle River

18. DeLong ER, DeLong DM, ClarkePearson DL (1988) Comparing the areas under two or more correlated receiver operating characteristic curves: a nonparametric approach. Biometrics 44:837-845

19. Shrout PE, Fleiss JL (1979) Intraclass correlations: uses in assessing rater reliability. Psychol Bull 86:420-428

20. Khemani RG, Newth CJ (2010) The design of future pediatric mechanical ventilation trials for acute lung injury. Am J Respir Crit Care Med 182:1465-1474

21. Ashbaugh DG, Bigelow DB, Petty TL et al (1967) Acute respiratory distress in adults. Lancet 2(7511):319-323
22. De Luca D, Piastra M, Tosi F et al (2012) Pharmacological therapies for pediatric and neonatal ALI/ARDS: an evidence-based review. Curr Drug Targets 13:906-916

23. De Luca D, Cogo P, Zecca E et al (2011) Intrapulmonary drug administration in neonatal and paediatric critical care: a comprehensive review. Eur Respir J 37:678-689

24. Kneyber MC, Brouwers AG, Caris JA, Chedamni S, Plötz FB (2008) Acute respiratory distress syndrome: is it under-recognized in the pediatric intensive care unit? Intensive Care Med 34:751-754

25. López-Fernández Y, Azagra AM, de la Oliva P et al (2012) Pediatric Acute Lung Injury Epidemiology and Natural History (PED-ALIEN) Network. Pediatric Acute Lung Injury Epidemiology and Natural History study: incidence and outcome of the acute respiratory distress syndrome in children. Crit Care Med 40:3238-3245

26. Angoulvant F, Llor J, Alberti C et al (2008) Inter-observer variability in chest radiograph reading for diagnosing acute lung injury in children. Pediatr Pulmonol 43:987-991

27. Rubenfeld GD, Caldwell E, Granton JT, Hudson LD, Matthay MA (1999) Interobserver variability in applying a radiographic definition for ARDS. Chest 116:1347-1353

28. Meade MO, Cook RJ, Guyatt GH et al (2000) Interobserver variation in interpreting chest radiographs for the diagnosis of acute respiratory distress syndrome. Am J Respir Crit Care Med 161:85-90

29. de Hemptinne Q, Remmelink M, Brimioulle $S$ et al (2009) ARDS: a clinic-pathologic confrontation. Chest 135:944-949

30. Thille AW, Esteban A, FernándezSegoviano P et al (2013) Comparison of the Berlin definition for acute respiratory distress syndrome with autopsy. Am J Respir Crit Care Med 187:761-767 